\title{
Stability of Kronecker products of irreducible characters of the symmetric group
}

\author{
Ernesto Vallejo ${ }^{1}$ \\ Instituto de Matemáticas \\ Universidad Nacional Autónoma de México \\ Area de la Inv. Cient. 04510 México, D.F. \\ evallejo@matem.unam.mx \\ Submitted: October 30, 1998; Accepted: September 6, 1999 \\ Primary classification 05E10, secondary classification 20C30
}

\begin{abstract}
F. Murnaghan observed a long time ago that the computation of the decompositon of the Kronecker product $\chi^{\left(n-a, \lambda_{2}, \ldots\right)} \otimes \chi^{\left(n-b, \mu_{2}, \ldots\right)}$ of two irreducible characters of the symmetric group into irreducibles depends only on $\bar{\lambda}=\left(\lambda_{2}, \ldots\right)$ and $\bar{\mu}=\left(\mu_{2}, \ldots\right)$, but not on $n$. In this note we prove a similar result: given three partitions $\lambda, \mu, \nu$ of $n$ we obtain a lower bound on $n$, depending on $\bar{\lambda}, \bar{\mu}, \bar{\nu}$, for the stability of the multiplicity $c(\lambda, \mu, \nu)$ of $\chi^{\nu}$ in $\chi^{\lambda} \otimes \chi^{\mu}$. Our proof is purely combinatorial. It uses a description of the $c(\lambda, \mu, \nu)$ 's in terms of signed special rim hook tabloids and Littlewood-Richardson multitableaux.
\end{abstract}

\section{Introduction.}

Let $\chi^{\lambda}$ denote the irreducible complex character of the symmetric group $\mathrm{S}(n)$ corresponding to the partition $\lambda$. For any three partitions $\lambda, \mu, \nu$ of $n$ we denote by

$$
c(\lambda, \mu, \nu):=\left\langle\chi^{\lambda} \otimes \chi^{\mu}, \chi^{\nu}\right\rangle
$$

the multiplicity of $\chi^{\nu}$ in the Kronecker product $\chi^{\lambda} \otimes \chi^{\mu}$.

F. Murnaghan observed in [6] that the computation of the decompositon of the Kronecker product $\chi^{\left(n-a, \lambda_{2}, \ldots\right)} \otimes \chi^{\left(n-b, \mu_{2}, \ldots\right)}$ into irreducibles depends only on $\bar{\lambda}=\left(\lambda_{2}, \ldots\right)$ and $\bar{\mu}=\left(\mu_{2}, \ldots\right)$, but not on $n$. He gave fifty eight formulas for decompositions of Kronecker products corresponding to the simplest choices of $\bar{\lambda}$ and $\bar{\mu}$. In fact, his formulas are valid for arbitrary $n$ only if one follows some rules to restore and discard disordered partitions appearing in them, see comment on [6, p.762]. In this note we prove a similar result: given three partitions $\lambda, \mu, \nu$ of $n$ we obtain a lower bound on $n$, depending on $\lambda, \mu, \nu$, for the stability of the coefficients $c(\lambda, \mu, \nu)$.

More precisely. Let $\bar{\lambda}=\left(\lambda_{2}, \ldots, \lambda_{p}\right), \bar{\mu}=\left(\mu_{2}, \ldots, \mu_{q}\right), \bar{\nu}=\left(\nu_{2}, \ldots, \nu_{r}\right)$ be partitions of positive integers $a, b, c$ respectively. For each $n \geq a+\lambda_{2}$ we consider the partition of $n, \lambda(n):=\left(n-a, \lambda_{2}, \ldots, \lambda_{p}\right)$. Similarly we define $\mu(n)$, and $\nu(n)$. Then we have

\footnotetext{
${ }^{1}$ Supported by DGAPA, UNAM IN103397
} 
Main Theorem. If $\bar{\nu}$ has one part and $\bar{\lambda}=\bar{\mu}$, let $m=\max \left\{\lambda_{2}+a+c, 2 c\right\}$; otherwise let $m=\max \left\{\lambda_{2}+a+c-1, \mu_{2}+b+c-1,2 c\right\}$. Then for all $n \geq m$

$$
c(\lambda(n), \mu(n), \nu(n))=c(\lambda(m), \mu(m), \nu(m)) .
$$

We note that $m$ is not symmetric on $\lambda, \mu, \nu$, but $c(\lambda, \mu, \nu)$ is. Therefore we may have three different choices for $m$ and we choose the smallest of the three. For example, consider partitions $(1),(2,1),(2,1)$. If we set $\bar{\lambda}=(1), \bar{\mu}=(2,1), \bar{\nu}=(2,1)$, then $a=1, b=3, c=3$ and $m=\max \{4,7,6\}=7$. However, if we set $\bar{\lambda}=(2,1)$, $\bar{\mu}=(2,1), \bar{\nu}=(1)$, then $a=3, b=3, c=1$ and $m=\max \{6,2\}=6$, and we get a sharper lower bound. This is the best possible, since $c((3,2,1),(3,2,1),(5,1))=2$ and $c((2,2,1),(2,2,1),(4,1))=1$.

We also note that the theorem does not always produce the best lower bound. For the partitions $(3,2),(2,2,1),(2,2)$ the lower bound given by the theorem is 11 . However, using SYMMETRICA [4], we obtained

$$
\begin{aligned}
& c((4,3,2),(4,2,2,1),(5,2,2))=12 \\
& c((5,3,2),(5,2,2,1),(6,2,2))=16 \\
& c((6,3,2),(6,2,2,1),(7,2,2))=16,
\end{aligned}
$$

which shows that the best lower bound is 10 .

The rest of this note is devoted to the proof of the theorem.

\section{Notation, definitions and known results}

In this section we fix the notation and record some definitions and results that will be used in the proof of the theorem.

Let $\lambda$ be a partition of $n$, in symbols $\lambda \vdash n$. We denote by $|\lambda|$ the sum of its parts, and by $\lambda^{\prime}$ its conjugate. We say that $\mu$ is contained in $\lambda$, in symbols $\mu \subseteq \lambda$, if $\mu_{i} \leq \lambda_{i}$ for all $i$. We use the notation $\lambda \unrhd \mu$ to indicate that $\lambda$ is greater or equal than $\mu$ in the dominance order. We denote by $\mathcal{P}(n)$ the diagram lattice, that is the set of partitions of $n$ together with the dominance order, see $[1,3,5,7]$.

Let $H$ be a subgroup of a group $G$. If $\chi$ is a character of $H$ we denote by $\operatorname{Ind}_{H}^{G}(\chi)$ the induction character of $\chi$. For any vector $\pi=\left(\pi_{1}, \ldots, \pi_{t}\right)$ of non-negative integers such that $\pi_{1}+\cdots+\pi_{t}=n$, let $\mathrm{S}(\pi)$ denote a Young subgroup of $\mathrm{S}(n)$ corresponding to $\pi$.

We denote by $\chi^{\lambda}$ the irreducible character of $\mathrm{S}(n)$ associated to $\lambda$, and by $\phi^{\lambda}=$ $\operatorname{Ind}_{\mathrm{S}(\lambda)}^{\mathrm{S}(n)}\left(1_{\lambda}\right)$ the permutation character associated to $\lambda$. They are related by the Young's rule

$$
\phi^{\mu}=\sum_{\lambda \unrhd \mu} K_{\lambda \mu} \chi^{\lambda},
$$


where $K_{\lambda \mu}$ is a Kostka number, that is, the number of semistandard tableaux of shape $\lambda$ and content $\mu$, see $[3,2.8 .5],[7, \S 2.11]$.

We will deal with two kinds of products of characters: Let $l, m$ be non-negative integers, and let $n=l+m$. Let $\chi_{1}$ be a character of $\mathrm{S}(l), \chi_{2}$ be a character of $\mathrm{S}(m)$, then

(i) $\chi_{1} \times \chi_{2}$ denotes the character of $\mathrm{S}(l) \times \mathrm{S}(m)$ given by $\chi_{1} \times \chi_{2}(\sigma, \tau)=\chi_{1}(\sigma) \chi_{2}(\tau)$.

(ii) $\chi_{1} \otimes \chi_{2}$ denotes, if $l=m$, the Kronecker product of $\chi_{1}$ and $\chi_{2}$, that is, the character of $\mathrm{S}(l)$ defined by $\chi_{1} \otimes \chi_{2}(\sigma)=\chi_{1}(\sigma) \chi_{2}(\sigma)$.

If $T$ is a tableau (a skew diagram filled with positive integers) there is a word $w(T)$ associated to $T$ given by reading the numbers of $T$ from right to left, in succesive rows, starting with the top row. Let $\pi=\left(\pi_{1}, \ldots, \pi_{t}\right)$ be a vector of positive integers such that $\pi_{1}+\cdots+\pi_{t}=n$. Let $\rho(i) \vdash \pi_{i}, 1 \leq i \leq t$. A sequence $T=\left(T_{1}, \ldots, T_{t}\right)$ of tableaux is called a Littlewood-Richardson multitableau of shape $\lambda$, content $(\rho(1), \ldots, \rho(t))$ and type $\pi$ if

(i) There exists a sequence of partitions

$$
0=\lambda(0) \subset \lambda(1) \subset \cdots \subset \lambda(t)=\lambda
$$

such that $|\lambda(i) / \lambda(i-1)|=\pi_{i}$ for all $1 \leq i \leq t$, and

(ii) for all $1 \leq i \leq t, T_{i}$ is a semistandard tableau of shape $\lambda(i) / \lambda(i-1)$ and content $\rho(i)$ such that $w\left(T_{i}\right)$ is a lattice permutation, see [3, 2.8.13], [5, I.9], [7, §4.9].

For each partition $\lambda$ of $n$ let $c_{(\rho(1), \ldots, \rho(t))}^{\lambda}$ denote the number of Littlewood-Richardson multitableaux of shape $\lambda$ and content $(\rho(1), \ldots, \rho(t))$. It follows by induction from the Littlewood-Richardson rule that

$$
\operatorname{Ind}_{\mathrm{S}(\pi)}^{\mathrm{S}(n)}\left(\chi^{\rho(1)} \times \cdots \times \chi^{\rho(t)}\right)=\sum_{\lambda \vdash n} c_{(\rho(1), \ldots, \rho(t))}^{\lambda} \chi^{\lambda} .
$$

Let

$$
\operatorname{lr}(\lambda, \mu ; \pi):=\left\langle\chi^{\lambda} \otimes \chi^{\mu}, \phi^{\pi}\right\rangle,
$$

then it follows from the Frobenius reciprocity theorem that

$$
\operatorname{lr}(\lambda, \mu ; \pi)=\sum_{\rho(1) \vdash \pi_{1}, \ldots, \rho(t) \vdash \pi_{t}} c_{(\rho(1), \ldots, \rho(t))}^{\lambda} c_{(\rho(1), \ldots, \rho(t))}^{\mu} .
$$

That is, $\operatorname{lr}(\lambda, \mu ; \pi)$ is the number of pairs $(S, T)$ of Littlewood-Richardson multitableaux of shape $(\lambda, \mu)$, same content, and type $\pi$.

Let $K_{n}=\left(K_{\lambda \mu}\right)$ be the Kostka matrix with rows and columns arranged in reverse lexicographical order, and let $K_{n}^{-1}=\left(K_{\lambda \mu}^{(-1)}\right)$ denote its inverse, see [5, I.6.5]. Then it follows from the Young rule (2) that

$$
\chi^{\nu}=\sum_{\pi \unrhd \nu} K_{\pi \nu}^{(-1)} \phi^{\pi} .
$$


Therefore from (1), (4) and (3) we obtain

\subsection{Proposition}

$$
c(\lambda, \mu, \nu)=\sum_{\pi \unrhd \nu} K_{\pi \nu}^{(-1)} \operatorname{lr}(\lambda, \mu ; \pi) .
$$

This formula gives, together with a result of Eg̃eciog̃lu and Remmel [2] (see Theorem $3.2)$, a combinatorial description of the numbers $c(\lambda, \mu, \nu)$. We will use it to get the stability of $c(\lambda, \mu, \nu)$ from the stability of $K_{\pi \nu}^{(-1)}$ and $\operatorname{lr}(\lambda, \mu ; \pi)$.

\section{Proof of the main theorem}

Let $\mathcal{P}(n)$ denote the diagram lattice, that is, the lattice of partitions of $n$ ordered under the dominance order, see $[1,3,5,7]$. For each partition $\nu$ of $n$, let $I_{\nu}$ denote the interval $\{\pi \vdash n \mid \nu \unlhd \pi \unlhd(n)\}$ in $\mathcal{P}(n)$.

3.1 Lemma. Let $n \geq 2 c$. Then the intervals $I_{\nu(n)}$ and $I_{\nu(2 c)}$ are isomorphic as posets.

Proof. For $\pi=\left(\pi_{1}, \ldots, \pi_{t}\right) \in I_{\nu(n)}$ we define $\widetilde{\pi}:=\left(\pi_{1}-(n-2 c), \pi_{2}, \ldots, \pi_{t}\right)$. It follows from the inequality $\pi \unrhd \nu(n)$ that $\tilde{\pi}$ is in $I_{\nu(2 c)}$. One can then easily verify that the map $\pi \mapsto \widetilde{\pi}$ is a poset isomorphism from $I_{\nu(n)}$ to $I_{\nu(2 c)}$.

In fact $2 c$ is the best lower bound: Choose $\bar{\nu}$ be any partition of $c$ with more than one part. Then $\nu(2 c)$ and $\nu(2 c-1)$ are well defined partitions, but $I_{\nu(2 c)} \approx I_{\nu(2 c-1)}$, because the partition $(c, c) \in I_{\nu(2 c)}$ has no corresponding partition in $I_{\nu(2 c-1)}$.

Next we prove a stability property for the numbers $K_{\lambda \mu}^{(-1)}$. For this we use a combinatorial interpretation of these numbers due to Eg̃eciog̃lu and Remmel [2]. Recall that a special rim hook tabloid T of shape $\mu$ and type $\lambda$ is a filling of the Ferrers' diagram of $\mu$ with rim hooks of sizes $\left\{\lambda_{1}, \ldots, \lambda_{p}\right\}$ such that each rim hook is special, that means, each rim hook has at least one box in the first column. The sign of a rim hook $H$ is $(-1)^{\mathrm{ht}(H)-1}$, where ht $(\mathrm{H})$ denotes, as usual, the height of the rim hook. And the sign of $T$ is defined as the product of the signs of the rim hooks of $T$, see [2, Section 2], [5, Ex. I.6.4] for details. Then

3.2 Theorem. (Ẽ̃geciog̃lu, Remmel [2])

$$
K_{\lambda \mu}^{(-1)}=\sum_{T} \operatorname{sign}(T)
$$

where the sum is over all special rim hook tabloids of type $\lambda$ and shape $\mu$.

From this we get the following two corollaries 
3.3 Corollary. Let $\bar{\nu}=\left(\nu_{2}, \ldots, \nu_{r}\right) \vdash c$, and $n \geq 2 c$. Then for all $\alpha(n), \beta(n)$ in $I_{\nu(n)}$ one has

$$
K_{\alpha(n) \beta(n)}^{(-1)}=K_{\alpha(2 c) \beta(2 c)}^{(-1)} .
$$

Proof. A sign preserving bijection between the set of special rim hook tabloids $T$ of type $\alpha(2 c)$ and shape $\beta(2 c)$ and the set of special rim hook tabloids $\widehat{T}$ of type $\alpha(n)$ and shape $\beta(n)$ is established in the following way: Let $H$ be the $\operatorname{rim}$ hook in $T$ which contains the last box from the first row. Then $H$ is of maximal length among the rim hooks in $T$. Let $\widehat{H}$ be the rim hook obtained from $H$ by adding $n-2 c$ boxes at the end of the first row, and let $\widehat{T}$ be obtained from $T$ by substituting $H$ by $\widehat{H}$. Since $H$ is of maximal length, then $\widehat{T}$ is a rim hook tabloid of type $\alpha(n)$. Clearly it has shape $\beta(n)$ and $\operatorname{sign}(T)=\operatorname{sign}(\widehat{T})$.

Another proof follows from [5, Ex. I.6.3].

3.4 Corollary. Let $\bar{\nu}=\left(\nu_{1}, \ldots, \nu_{r}\right), \bar{\pi}=\left(\pi_{2}, \ldots, \pi_{t}\right) \vdash c$, and suppose $r>2$. Then

$$
K_{\pi(2 c) \nu(2 c)}^{(-1)}=K_{\bar{\pi} \bar{\nu}}^{(-1)}
$$

Since the sum of the entries of any column of the inverse Kostka matrix (with the obvious exception of the first one) is zero, then it follows

3.5 Corollary. Let $m=2 c$, and suppose $r>2$. Then

$$
\sum_{\pi(m) \unrhd \nu(m),} K_{\pi(m)_{1}=c}^{(-1)} K_{\pi(m) \nu(m)}^{(m)}=0 .
$$

Let denote $\operatorname{LR}(\lambda(n), \mu(n) ; \nu(n))$ the set of pairs $(S, T)$ of Littlewood-Richardson multitableaux of shape $(\lambda(n), \mu(n))$, same content and type $\nu(n)$.

3.6 Lemma. Let $m=\max \left\{\lambda_{2}+a, \mu_{2}+b, \nu_{2}+c\right\}$. Then for all $n \geq m$ there is an injective map

$$
\Phi: \operatorname{LR}(\lambda(m), \mu(m) ; \nu(m)) \longrightarrow \operatorname{LR}(\lambda(n), \mu(n) ; \nu(n)) .
$$

Proof. Let $(S, T) \in \operatorname{LR}(\lambda(m), \mu(m) ; \nu(m))$. Let $\widehat{S}$ be obtained from $S$ by adding $n-m 1$ 's at the end of the first row of $S_{1}$, and shifting $n-m$ places to the right the remaining 1's belonging to the tableaux $S_{2}, \ldots S_{r}$. Let $\widehat{T}$ be defined in a similar way. Then $(\widehat{S}, \widehat{T})$ belongs to $\operatorname{LR}(\lambda(n), \mu(n) ; \nu(n))$, and the map $\Phi(S, T):=(\widehat{S}, \widehat{T})$ is injective. 
3.7 Proposition. Let $m=\max \left\{\lambda_{2}+a+c-1, \mu_{2}+b+c-1, \nu_{2}+c\right\}$ if $\bar{\lambda} \neq \bar{\mu}$, and $m=\max \left\{\lambda_{2}+a+c, \nu_{2}+c\right\}$ if $\bar{\lambda}=\bar{\mu}$. Then for all $n \geq m$

$$
\operatorname{lr}(\lambda(n), \mu(n) ; \nu(n))=\operatorname{lr}(\lambda(m), \mu(m) ; \nu(m)) .
$$

Proof. We show that under our hypothesis, we can define a map

$$
\Psi: \operatorname{LR}(\lambda(n), \mu(n) ; \nu(n)) \longrightarrow \operatorname{LR}(\lambda(m), \mu(m) ; \nu(m))
$$

inverse to $\Phi$. Let $(S, T)$ be in $\operatorname{LR}(\lambda(n), \mu(n) ; \nu(n))$, and let $(\rho(1), \ldots, \rho(r))$ be the common content of $S$ and $T$. We define $\widetilde{\rho(1)}:=\left(\rho(1)_{1}-(n-m), \rho(1)_{2}, \ldots, \rho(1)_{u}\right)$, where $u$ is the length of $\rho(1)$. Note that $\rho(1) \subseteq \lambda(n)$ and that $|\lambda(n) / \rho(1)|=c$. Then, if $\bar{\lambda}=\bar{\mu}$, we have that $\rho(1)_{1} \geq \lambda(n)_{1}-c=n-a-c \geq n-m+\lambda_{2}$. And, if $\bar{\lambda} \neq \bar{\mu}$, we have that $\rho(1)_{1} \geq \lambda(n)_{1}-(c-1)=n-a-(c-1) \geq n-m+\lambda_{2}$. Therefore, in both cases, $\rho(1)_{1}-(n-m) \geq \lambda_{2} \geq \rho(1)_{2}$, and $\widetilde{\rho(1)}$ is a partition of $m-c=\nu(m)_{1}$. Let $\widetilde{S}$ be obtained from $S$ by deleting the first $(n-m)$ 1's in the first row and shifting to the left the remaining numbers $n-m$ places. In this way $\widetilde{S}$ is a multitableau of shape $\lambda(m)$, content $(\widetilde{\rho(1)}, \rho(2), \ldots, \rho(r))$ and type $\nu(m)$. Moreover, since $\rho(1)_{1}-(n-m) \geq \lambda_{2}$, $\widetilde{S}$ is a Littlewood-Richardson multitableau. We define in a similar way a LittlewoodRichardson multitableau $\widetilde{T}$ of shape $\mu(m)$, same content as $\widetilde{S}$ and type $\nu(m)$. It is straightforward to check that the map $(S, T) \mapsto(\widetilde{S}, \widetilde{T})$ yields the inverse of $\Phi$.

3.8 Corollary. Let $m$ be defined as in Proposition 3.7. Let $\pi(m)=\left(m-e, \pi_{2}, \ldots, \pi_{t}\right)$ be in $I_{\nu(m)}$. Then for all $n \geq m$

$$
\operatorname{lr}(\lambda(n), \mu(n) ; \pi(n))=\operatorname{lr}(\lambda(m), \mu(m) ; \pi(m)) .
$$

The main theorem now follows from Proposition 2.1, Corollaries 3.3 and 3.8, either if $\bar{\lambda} \neq \bar{\mu}$, or if $\bar{\lambda}=\bar{\mu}$ and $r=2$. It remains to prove it in the case $\bar{\lambda}=\bar{\mu}$ and $r>2$.

3.9 Lemma. Let $m=\max \left\{\lambda_{2}+a+c-1,2 c\right\}, \pi(m)=\left(m-c, \pi_{2}, \ldots, \pi_{t}\right)$ be in $I_{\nu(m)}$, and suppose $r>2$. Then for all $n>m$

$$
\operatorname{lr}(\lambda(n), \lambda(n) ; \pi(n))=\operatorname{lr}(\lambda(m), \lambda(m) ; \pi(m))+1
$$

Proof. Let $(S, T)$ be in $\operatorname{LR}(\lambda(n), \lambda(n) ; \pi(n))$, and let $(\rho(1), \ldots, \rho(t))$ be the common content of $S$ and $T$. Then, as in the proof of Proposition 3.7, we have that $\rho(1)_{1} \geq$ $n-a-c$. If $\rho(1)_{1}>n-a-c$, then $\rho(1)_{1} \geq n-a-(c-1) \geq n-m+\lambda_{2}$. Again, as in the proof of Proposition 3.7, there exists $(\widetilde{S}, \widetilde{T}) \in \operatorname{LR}(\lambda(m), \lambda(m) ; \pi(m))$, such that $\Phi(\widetilde{S}, \widetilde{T})=(S, T)$. If $\rho(1)_{1}=n-a-c$, then $\lambda(n) / \rho(1)=(c)$. In this situation, there is exactly one Littlewood-Richardson multitableau $R$ of shape $\lambda(n)$ and type $\pi(n)$. 
It has content $\left(\lambda(n) /(c),\left(\pi_{2}\right), \ldots,\left(\pi_{t}\right)\right)$. Therefore the pair $(R, R)$ is the only one in $\operatorname{LR}(\lambda(n), \lambda(n) ; \pi(n))$ which is not in the image of $\Phi$. The claim follows.

3.10 Corollary. Let $m=\max \left\{\lambda_{2}+a+c-1,2 c\right\}$, and suppose $r>2$. Then for all $n>m$

$$
c(\lambda(n), \lambda(n), \nu(n))=c(\lambda(m), \lambda(m), \nu(m))
$$

Proof. By Proposition 2.1 it is enough to prove

$$
\sum_{\pi(n) \unrhd \nu(n)} K_{\pi(n) \nu(n)}^{(-1)} \operatorname{lr}(\lambda(n), \lambda(n) ; \pi(n))=\sum_{\pi(m) \unrhd \nu(m)} K_{\pi(m) \nu(m)}^{(-1)} \operatorname{lr}(\lambda(m), \lambda(m) ; \pi(m)) .
$$

Note that if $\pi(n)=\left(n-e, \pi_{2}, \ldots, \pi_{t}\right)$ and $e<c$, then by Proposition 3.7

$$
\operatorname{lr}(\lambda(n), \lambda(n) ; \pi(n))=\operatorname{lr}(\lambda(m), \lambda(m) ; \pi(m))
$$

and if $e=c$, then by Lemma 3.9

$$
\operatorname{lr}(\lambda(n), \lambda(n) ; \pi(n))=\operatorname{lr}(\lambda(m), \lambda(m) ; \pi(m))+1 .
$$

The claim now follows from Corollaries 3.3 and 3.5.

\section{References}

[1] T. Brylawski, The lattice of integer partitions, Discrete Math. 6 (1973), 201-219.

[2] Ö. Eg̃eciog̃lu and J.B. Remmel, A combinatorial interpretation of the inverse Kostka matrix, Linear and Multilinear Algebra 26 (1990), 59-84.

[3] G.D. James and A. Kerber, The representation theory of the symmetric group, Encyclopedia of mathematics and its applications, Vol. 16, Addison-Wesley, Reading, Massachusetts, 1981.

[4] A. Kerber and A. Kohnert, SYMMETRICA 1.0, October 1994, Univ. of Bayreuth.

[5] I.G. Macdonald, Symmetric functions and Hall polynomials, 2nd. edition, Oxford Univ. Press, Oxford, 1995.

[6] F.D. Murnaghan, The analysis of the Kronecker product of irreducible representations of the symmetric group, Amer. J. Math. 60 (1938), 761-784.

[7] B.E. Sagan, The symmetric group, Wadsworth \& Brooks/Cole, Pacific Grove, California, 1991. 